INPLASY

PROTOCOL

To cite: Han et al. Effect of Electroacupuncture on Glucose and Lipid Metabolism in Type 2 Diabetes: A protocol for Systematic Review and Meta-Analysis. Inplasy protocol 202180008. doi: 10.37766/inplasy2021.8.0008

Received: 03 August 2021

Published: 03 August 2021

Corresponding author: Yiran Han

miaran0910@163.com

Author Affiliation: Changchun University of Chinese Medicine, Changchun, Jilin Province, PR

China.

Support: NSFC.

Review Stage at time of this submission: Preliminary searches.

Conflicts of interest:

None declared.

\section{Effect of Electroacupuncture on Glucose and Lipid Metabolism in Type 2 Diabetes: A protocol for Systematic Review and Meta-Analysis}

\author{
Han, Y1; Lu, Z²; Meng, M³; Wang, H; Ting, P5; Gao, T6; Liu, M7.
}

Review question / Objective: It has been unclear whether electoacupuncture therapy improves clinical outcomes in type 2 diabetic patients. This study aimed to summarize and assess the effect of electroacupuncture as supplemental method to treat type 2 diabetes patients from clinical trials and provide more options on fasting plasma glucose (FPG), glycated hemoglobin (HbA1c), fasting insulin, and lipid profile for clinicians and patients to treat obese type 2 diabetes. Condition being studied: Obese type 2 diabetes. Language is limited to Chinese and English. Clinical randomized controlled trials related to acupuncture for obese type 2 diabetes will be included in the study.

INPLASY registration number: This protocol was registered with the International Platform of Registered Systematic Review and Meta-Analysis Protocols (INPLASY) on 03 August 2021 and was last updated on 03 August 2021 (registration number INPLASY202180008).

\section{INTRODUCTION}

Review question / Objective: It has been unclear whether electoacupuncture therapy improves clinical outcomes in type 2 diabetic patients. This study aimed to summarize and assess the effect of electroacupuncture as supplemental method to treat type 2 diabetes patients from clinical trials and provide more options on fasting plasma glucose (FPG), glycated hemoglobin (HbA1c), fasting insulin, and lipid profile for clinicians and patients to treat obese type 2 diabetes.

Condition being studied: Obese type 2 diabetes. Language is limited to Chinese and English. Clinical randomized controlled 
trials related to acupuncture for obese type 2 diabetes will be included in the study.

\section{METHODS}

Search strategy: We will electronically search PubMed, Medline, Embase, Web of Science, the Cochrane Central Register of Controlled Trials, China National Knowledge Infrastructure, Chinese Biomedical Literature Database, Chinese Scientific Journal Database, and Wan-Fang Database from the date of creation to December 2022. In addition, we will manually retrieve other resources including the reference lists of identified publications, conference articles, and gray literature.

Participant or population: Type 2 diabetes patients.

Intervention: Interventions can be any type of electropuncture.

Comparator: Multiple control interventions will be included: no treatment, placebo and other interventions (e.g., cupping therapy, drugs, and physical interventions, moxibustion).

Study designs to be included: Randomized controlled clinical trials and nonrandomized controlled trials will be included.

Eligibility criteria: The diagnosis of T2DM is in accordance with who's diagnostic criteria for diabetes in 1999.Mean-while, the participants who are included have no limitation of age, sex, region, citizenship, and nationality. Cases related to serious diseases, pregnancy, and drug-induced obesity areexcluded.

Information sources: We will electronically search PubMed, Medline, Embase, Web of Science, the Cochrane Central Register of Controlled Trials, China National Knowledge Infrastructure, Chinese Biomedical Literature Database, Chinese Scientific Journal Database, and Wan-Fang Database from the date of creation to December 2022. In addition, we will manually retrieve other resources including the reference lists of identified publications, conference articles, and gray literature.

Main outcome(s): (1)fasting plasma glucose (FPG), (2)glycated hemoglobin (HbA1c), (3)fasting insulin, (4) lipid profile.

Additional outcome(s): (1) weight loss (kg), (2) homeostatic model assessment of insulin resistance (HOMA-IR) level,(3) dropout, (4) side effects, (5) rebound.

Data management: Two authors will independently extract data. Any disagreement will be resolved by discussion until consensus is reached or by consulting a third author. The following data will be extracted: author, year of publication, country where the study was conducted, study period, original inclusion criteria, total number of people included in the study, intervention measures, outcome indicators, adverse reactions, among others.

Quality assessment / Risk of bias analysis: The literature quality of this study was evaluated by the bias risk table proposed by Cochrane collaborative network. The risk table includes 6 items: random sequence generation mode, whether to use allocation concealment, whether to blind the subjects and intervention providers, whether to blind the results evaluators, whether the results data are complete, whether to select the results report and other bias sources. The criteria used to assess the risk of bias are "low risk," "high risk," and "unclear." In this process, 2 evaluators independently evaluate the methodological quality. In case of disagreement, the third author would be intervened.

Strategy of data synthesis: RevMan V.5.3.5. software will be used for all statistical analyses. We decided to use either a fixedeffects or random-effects model based on the heterogeneity levels of the included studies. If no substantial statistical heterogeneity is detected, the data 
synthesis will be processed using the fixedeffects model, and if substantial statistical heterogeneity is detected, the data synthesis will be performed using the random-effects model.The terms for searching were: ('electoacupuncture OR electropuncture) AND ('supple intervention') AND ('type 2 diabetes mellitus') AND (glucose metabolism OR Lipid metabolism) AND (randomized OR blind OR placebo OR meta-analysis). We also attempted to contact the investigators if their clinical end-points were not reported.

Subgroup analysis: To eliminate heterogeneity as much as possible, sensitivity analysis or subgroup analysis is applied to explore the source of heterogeneity. If one considers the impact of multiple covariates, a meta-regression analysis is applied to explore the source of heterogeneity.

Sensitivity analysis: In the direct comparison. if there is a large heterogeneity and the number of studies included is enough, we will use the method of meta regression for sensitivity analysis, otherwise we will exclude the studies one by one for sensitivity analysis.

Country(ies) involved: China.

Keywords: acupuncture; type 2 diabetes mellitus; meta-analysis; systematic review.

Contributions of each author:

Author 1 - Yiran Han.

Author 2 - Zeyuan Lu.

Author 3 - Meng Meng.

Author 4 - Heran Wang.

Author 5 - Pan Ting.

Author 6 - Tianjiao Gao.

Author 7 - Mingjun Liu. 